\title{
Reference Trajectory Based Quasi-Sliding Mode with Event-Triggered Control
}

\author{
Katarzyna Adamiak* (D) and Andrzej Bartoszewicz (1D \\ Institute of Automatic Control, Łódź University of Technology, 18 Bohdana Stefanowskiego Street, \\ 90-537 Łódź, Poland; andrzej.bartoszewicz@p.lodz.pl \\ * Correspondence: katarzyna.adamiak@p.lodz.pl
}

check for updates

Citation: Adamiak, K.;

Bartoszewicz, A. Reference Trajectory Based Quasi-Sliding Mode with Event-Triggered Control. Energies 2021, 14, 7236. https://doi.org/ $10.3390 /$ en14217236

Academic Editor: Teuvo Suntio

Received: 22 September 2021

Accepted: 28 October 2021

Published: 2 November 2021

Publisher's Note: MDPI stays neutral with regard to jurisdictional claims in published maps and institutional affiliations.

Copyright: (c) 2021 by the authors. Licensee MDPI, Basel, Switzerland. This article is an open access article distributed under the terms and conditions of the Creative Commons Attribution (CC BY) license (https:// creativecommons.org/licenses/by/ $4.0 /)$.

\begin{abstract}
The study presents a novel event-triggered quasi-sliding mode control algorithm for linear discrete time systems. The problem is divided into two main parts. Firstly, the sliding mode control of perturbed discrete time systems is considered. In order to limit the impact of external disturbances to one sampling step only, a reference trajectory-based control law is introduced. The proposed control method drives the system's representative point to an a priori designed reference position in each control step, thus minimizing the influence of disturbance and improving the robustness. Moreover, the reference trajectory is generated according to a novel reaching law, which ensures the nonswitching movement within the quasi-sliding mode band. In the latter part of the study, the proposed control strategy is supplemented with an event-triggering algorithm. In the modified strategy the control signal is only updated when a certain triggering condition occurs. Therefore, the need for communication between system elements is reduced. As follows, the delays in the digital control process may be reduced as well, without compromising the system's robustness.
\end{abstract}

Keywords: control design; discrete time systems; event-triggering; reaching law approach; sliding mode control; reference trajectory generator

\section{Introduction}

Sliding mode design has been drawing the attention of the control engineering society for decades now. The idea first appeared in Russian literature concerning variable structure systems in the 1960-ties [1]. However, the concept of sliding mode had not gained worldwide interest until the late 1970-ties, when the English-written paper of Utkin was first published [2]. The basic concept introduced a two-stage control design process. First, a sliding manifold is selected. Its choice is crucial for the stability of the control system, so it must contain the desired steady state position. Afterwards, a nonlinear control law should be designed so that it restricts the system's state to the selected sliding hyperplane. Therefore, the controller always drives the representative point onto the sliding surface, regardless of matched modelling uncertainties and external disturbances [3].

Gradually, the sliding mode control concept has been gathering more and more followers from all over the world [4-9]. The unique properties of the sliding mode have resulted in numerous practical applications [9], including those for electromechanical systems $[10,11]$. However, with the digital implementation of the strategy, a new problem emerged. Sampling in the input and output channels causes inevitable delays in communication between the elements of the system. Consequently, the frequency of the control signal updates becomes limited, and the system's state may deviate from the sliding manifold. Switching the control signal with high frequency also leads to the chattering phenomenon, where the system starts to oscillate [12]. Such a behaviour is not only inefficient and causes unnecessary energy losses but also may result in damaging some electromechanical components. These observations triggered the study of discrete time sliding mode. The so-called quasi-sliding mode, first studied by Milosavljević [13], exists if the representative point of the system is driven to the vicinity of the sliding surface 
represented by the quasi-sliding mode band and remains within this band for the rest of the control process. Later works have presented two interpretations of this definition. Some authors assumed that the representative point should cross the sliding surface in each control step for the quasi-sliding motion to occur [14]. Others only required that, once the band has been reached, the representative point never leaves it again [15-17]. Nevertheless, the movement, divided into a reaching phase and a sliding phase, could be easily obtained using one of numerous reaching laws, such as [18-22]. However, regardless of the utilised reaching function, the discrete time sliding mode always bears the impact of external disturbances and modelling uncertainties, as the control may only be switched at the discrete time instants. The measure of the system's robustness to those perturbations is the width of the resulting quasi-sliding mode band.

In order to increase the system's robustness to disturbances and model uncertainties, in this study, we apply a reference trajectory following a sliding mode control scheme. The idea originates from the model following control applied to both continuous and discretised systems $[23,24]$. However, in our concept, an actual mathematical model of the considered system is not required. Instead, we generate the desired sliding variable profile with a trajectory generator. As the sliding variable combines all state variables of the considered system, we notice that obtaining its reference trajectory is sufficient to implement a trajectory-following control scheme. Moreover, the trajectory generator utilises a novel nonswitching type reaching law. Next, a trajectory tracking control law is used to drive the system's representative point to the reference position. Unlike most of the known reaching law-based control schemes, which use the sliding variable to obtain the control signal, our approach uses the reference sliding variable value to calculate the control. As the reference is independent of any perturbations, the impact of disturbances on the actual system is limited to one discrete instant. In other words, in some step $k$, the position of the system bears the influence of the last disturbance value from step $k-1$, whereas in the conventional sliding mode, the sliding variable holds the impact of all perturbations from steps $0,1,2, \ldots, k-1$ and passes it on to the upcoming instants. Therefore, by limiting the disturbance influence, the model following approach increases the system's robustness, as shown in [24]. Moreover, considering the problem of the energetical efficiency of the control system, a nonswitching type of motion around the sliding plane is enforced. Therefore, the energy consumption in the sliding phase is significantly reduced compared to switching type quasi-sliding mode.

In the further part of the paper, the presented control strategy is supplemented with an event-triggering scheme. The event-triggered sliding mode is one of the recent trends in variable structure control [25-29]. This strategy assumes updating the control signal only when a certain stability condition is violated or, in other words, when a selected triggering condition is met. Therefore, the need for communication between sensor and actuator ends of the control system is minimised, which reduces delays in the digital control process. Such an advantage is especially important in spatially distributed control systems, such as communication networks, where delays play a significant role in worsening the robustness. In this paper, we combine the trajectory tracking quasi-sliding mode with an event-triggered control law. We aim to update the control signal only when the representative point of the system deviates from the reference position by more than a selected stability margin. Therefore, the stability will be preserved along with a more efficient usage of the system's resources.

The considerations of this paper are organised as follows. Section 2 states the dynamic problem we are discussing. Next, Section 3 presents the reference trajectory-based quasisliding mode control and Section 4 introduces the event-triggered approach. Finally, Section 5 provides a simulation example and Section 6 includes our conclusions and closing remarks. 


\section{Problem Statement}

This study considers the design of discrete time sliding mode control for the following linear system

$$
\boldsymbol{\eta}(k+1)=\boldsymbol{\Phi} \boldsymbol{\eta}(k)+\boldsymbol{\Gamma}[u(k)+f(k)],
$$

where $\eta(k)$ is the state vector of the system and $\eta \in \mathbb{R}^{n}, n$ is the order of the system, $u(k)$ represents a control signal, and $u \in \mathbb{R}, f(k)$ denotes matched external disturbance and $\Phi$ and $\Gamma$ are the system's state matrix and control distribution vector of appropriate dimensions. The system's initial position is denoted by $\boldsymbol{\eta}_{0}=\boldsymbol{\eta}(0)$, and the objective is to drive all the state variables to zero. The disturbance $f(k)$, acting through the control channel, is unknown. However, in order to design a control scheme, we assume it is upper and lower bounded, so that

$$
|f(k)| \leq f_{\max }
$$

always holds and $f_{\max }$ is a known real positive constant.

This study proposes to control the system Equation (1) with a quasi-sliding mode control scheme. For that purpose, we introduce a sliding plane

$$
s(k)=c \eta(k)=0
$$

where $c$ is an $1 \times n$ vector. The value of the emerging sliding variable $s(k)$ describes the current distance between the system's representative point and the plane Equation (3). The design of the sliding plane becomes a proper selection of vector $c$, which determines the steady-state behaviour of the sliding mode controlled system. The sliding variable bears the impact of the external disturbance $\mathrm{c} \Gamma f(k)$, which for simplicity is further denoted by $D(k)$. As results from Equation (2), the following holds for $D(k)$

$$
|D(k)| \leq D_{\max }=\left|\mathbf{c} \Gamma f_{\max }\right|
$$

where $D_{\max }$ is a real positive constant. Having introduced all the necessary notations, we will next propose a reference trajectory based sliding mode control algorithm.

\section{Reference Trajectory Based Quasi-Sliding Mode Control}

This section introduces a trajectory tracking sliding mode control algorithm for the perturbed plant Equation (1). In the first step, we generate the demand trajectory represented by the reference sliding variable. Its evolution is obtained with a novel nonswitching type reaching law, which ensures monotonic convergence to the preselected sliding manifold. Next, the actual system will be steered to the reference position with a trajectory tracking control law. It will be shown that, in spite of the presence of external disturbances, convergence and stability of the system are preserved.

\subsection{Reference Trajectory Generation}

Before we design the control signal for the original system Equation (1), we need to generate its desired trajectory, which will serve as a reference in this control scheme. For that purpose we define a reference sliding variable $s_{r}(k)$, with the initial value $s_{r}(0)=s(0)=c \eta_{0}$. As the sliding variable $s(k)$ combines all the state variables of the system, we conclude that its reference profile is sufficient for a model following the control scheme. The evolution of the reference sliding variable is obtained with a novel reaching law

$$
s_{r}(k+1)=\left\{\begin{array}{cc}
(\alpha+\beta) s_{r}(k)-\alpha \beta s_{r}(k-1) & \text { for } \alpha \beta\left|s_{r}(k-1)\right|>2 D_{\max } \\
0 & \text { for } \alpha \beta\left|s_{r}(k-1)\right| \leq 2 D_{\max }
\end{array}\right.
$$

where parameters $\alpha$ and $\beta$ are real positive constants, chosen so that

$$
0<\alpha, \beta<1 \text { and } \alpha+\beta \leq 1
$$


The reaching law Equation (5) is formulated in two terms. When the representative point is far from the sliding surface, i.e., when $s_{r}(k) \gg 0$, the first term of the reaching law becomes dominant, so we get

$$
s_{r}(k+1)=(\alpha+\beta) s_{r}(k)-\alpha \beta s_{r}(k-1) .
$$

It may be easily proved that the function Equation (7) provides monotonic convergence to the sliding plane Equation (3). Let us consider the reference sliding variable value in two consequents steps $k$ and $k-1$, denoting $s_{r}(k)$ with $x_{1}(k)$ and $s_{r}(k-1)$ with $x_{2}(k)$. With this notation, from Equation (7) we get

$$
\begin{aligned}
& x_{1}(k+1)=(\alpha+\beta) x_{1}(k)-\alpha \beta x_{2}(k) \\
& x_{2}(k+1)=x_{1}(k) .
\end{aligned}
$$

It may be observed that the Equations in (8) describe a second order, linear, discrete time system and they may be transformed to the following state equation

$$
\left[\begin{array}{l}
x_{1}(k+1) \\
x_{2}(k+1)
\end{array}\right]=\left[\begin{array}{cc}
\alpha+\beta & -\alpha \beta \\
1 & 0
\end{array}\right]\left[\begin{array}{l}
x_{1}(k) \\
x_{2}(k)
\end{array}\right]
$$

The characteristic polynomial of system Equation (9) is

$$
M(z)=(z-\alpha)(z-\beta),
$$

which shows that the eigenvalues of the system are $z_{1}=\alpha$ and $z_{2}=\beta$. As, according to Equation (6), both $\alpha$ and $\beta$ are positive and smaller than 1, we conclude that the eigenvalues are located within the unit circle. Therefore, for any $k \geq 0$, the absolute value of the reference sliding variable is monotonically decreasing, so

$$
\left|s_{r}(k+1)\right|<\left|s_{r}(k)\right|
$$

Moreover, the rate of the decline is governed by the choice of $\alpha$ and $\beta$. When the parameter values are bigger, so the poles of system Equation (9) are located further from the origin of the complex plane, then the value of $s_{r}(k)$ decreases slower. On the other hand, when the poles of Equation (9) are closer to the origin, the rate of the decline is faster. Therefore, while choosing the control parameters the designer decides about the convergence pace. Considering Equation (6), the rate of change of the reference, denoted with $\Delta s_{r}$, satisfies

$$
\Delta s_{r}=\left|s_{r}(k)-s_{r}(k+1)\right|=\underbrace{[1-(\alpha+\beta)]}_{\langle 0 ; 1\rangle}\left|s_{r}(k)\right|+\underbrace{\alpha \beta\left|s_{r}(k-1)\right|}_{>2 D_{\max }} .
$$

Thus

$$
\Delta s_{r}>2 D_{\max }
$$

The above proves that the reference sliding variable monotonically converges to the vicinity of the sliding surface Equation (3). Consequently, there always exists such a moment $k_{0}$ when

$$
\alpha \beta s_{r}\left(k_{0}-1\right) \leq 2 D_{\max }
$$

and for any $k \geq k_{0}$ the second term of the reaching law Equation (5) acts. Consequently, for any $k \geq k_{0}+1$ the reference sliding variable equals zero, i.e.,

$$
s_{r}(k)=0 \text { for } k \geq k_{0}+1 \text {. }
$$




\subsection{Quasi-sliding Mode Control Law}

After the reference sliding variable evolution has been obtained, we may proceed to design the control signal for the original system Equation (1), subject to matched external disturbances. The task of the controller is to drive the system's representative point to the reference position with the accuracy of the disturbance. This is ensured with the following reaching law

$$
s(k+1)=s_{r}(k+1)+D(k) .
$$

Taking into account Equations (1) and (16) the control signal required to drive the system to the sliding surface Equation (3) is

$$
u(k)=-(\mathbf{c} \boldsymbol{\Gamma})^{-1}\left[\boldsymbol{c} \boldsymbol{\Phi} \boldsymbol{\eta}(k)-s_{r}(k+1)\right] .
$$

Considering boundedness of the disturbance Equation (4) and the reaching law Equation (16) the above signal ensures that

$$
\left|s_{r}(k)\right|-D_{\max } \leq|s(k)| \leq\left|s_{r}(k)\right|+D_{\max }
$$

for any $k \geq 0$. In the same manner, we may express the bounds of $s(k+1)$. We get

$$
\left|s_{r}(k+1)\right|-D_{\max } \leq|s(k+1)| \leq\left|s_{r}(k+1)\right|+D_{\max }
$$

Now, in order to prove the convergence of the system to the vicinity of the sliding surface, let us consider the rate of change of the sliding variable. Taking into account the worst cases for Equations (18) and (19), for the rate of change of the sliding variable we obtain

$$
\left|s_{r}(k)-s_{r}(k+1)\right|-2 D_{\max } \leq|s(k)|-|s(k+1)| \leq\left|s_{r}(k)-s_{r}(k+1)\right|+2 D_{\max } .
$$

Moreover, taking into account the evolution of the reference Equation (5) in the first stage of motion and its rate of change Equation (13), we get

$$
\Delta s_{r}-2 D_{\max } \leq|s(k)|-|s(k+1)| \leq \Delta s_{r}+2 D_{\max } .
$$

Remembering Equation (13), we conclude that the absolute value of the sliding variable decreases in each consecutive time instant. Therefore, the sliding variable of the actual system $s(k)$ converges to the vicinity of the sliding surface even in the worst case of external disturbances. Finally, for any $k \geq k_{0}+1$, the reference is equal to zero. As follows directly from Equation (16), for any $k \geq k_{0}+1$, the sliding variable $s(k)$ belongs to $\pm D_{\max }$ vicinity of the sliding plane, i.e.,

$$
|s(k)| \leq D_{\max } \text { for } k \geq k_{0}+1 .
$$

As presented above, the proposed control strategy drives the system's representative point to the $\pm D_{\max }$ vicinity of the selected sliding manifold in finite time. Application of the reference sliding variable based scheme ensures that the control signal is based on the demand sliding variable. Thus, in step $k$ the sliding variable is only influenced by the last disturbance $D(k-1)$. On the contrary, in the conventional quasi-sliding mode design, the control is calculated using the reaching law and the previous sliding variable values. Thus, the sliding variable $s(k)$ bears the influence of all disturbance values $D(0), D(1), \ldots, D(k-1)$. Consequently, the application of the reference sliding variable directly improves the robustness of the system. It is also worth mentioning that the novel reaching law Equation (5) provides a non-switching type of movement for the reference and, thus, for the actual sliding variable as well. In the quasi-sliding phase, the representative point of the system moves within the quasi-sliding mode band, but it does not have to cross the sliding plane in each step. Elimination of the switching requirement directly results in a reduction of the control effort in the sliding phase. As follows, the proposed 
strategy ensures significant saving of energy, which is nowadays an important factor in electronic control design.

\section{Event-Triggered QSM Control}

The quasi-sliding mode control scheme presented in the previous section requires the control signal to be updated in each consecutive time instant $k, k+1, k+2, \ldots$. Although this periodic approach ensures monotonic convergence, stability, and improved robustness of the system, updating the control value in every sampling instant may turn out not to be economical due to excessive energy consumption, computational expenses, and delays. Therefore, in this section, we modify the presented strategy by adding an event-triggering algorithm.

\subsection{Triggering Condition}

So far, the control signal value was updated at each discrete time instant $k$. Let us denote these instants with the sequence $\{k\}$, where $k=1,2,3, \ldots$. From now on we aim to update the control signal only when a certain stability criterium is violated or, in other words, when a triggering condition is met. As the system Equation (1) is defined in the discrete domain, the triggering condition must be calculated at all discrete time instants $k$. However, the control must only be updated at those instants $j$, when the triggering condition is satisfied. Therefore, let us denote the triggering instants series with $\{j\}$, where $j=1,2,3, \ldots$ and the time gap between the consecutive instants $j$ and $j+1$ varies. Aperiodic updates of the control signal may cause the state trajectory error to increase between the consecutive instants $j$ and $j+1$. Thus, let us denote the state error introduced by the event-triggered control scheme with

$$
\boldsymbol{e}(k)=\boldsymbol{\eta}(j)-\boldsymbol{\eta}(k),
$$

where $j$ is the instant when the control signal was last updated. To ensure the stability of the closed-loop system, the state error expressed with Equation (23) must remain bounded. Therefore, for any $k \geq 0$ the following stability condition must hold

$$
|c \Phi e(k)|<\varepsilon,
$$

where $\varepsilon$ is a selected admissible bound and $\varepsilon>0$. The parameter $\varepsilon$ will be used to determine the triggering instants. We aim to update the control signal each time the stability condition Equation (24) is violated. Therefore, the triggering instants are defined as

$$
j+1=\inf \{k: k>j \wedge|c \Phi e(k)| \geq \varepsilon\} .
$$

Consequently, at any time instant $k$ between the consecutive control updates $j$ and $j+1$, the control remains constant. Therefore, the control signal previously described with Equation (17) now becomes

$$
u(k)=\left\{\begin{array}{cccc}
-(\boldsymbol{c} \Gamma)^{-1}\left[\boldsymbol{c} \boldsymbol{\Phi} \boldsymbol{\eta}(k)-s_{r}(k+1)\right] & \text { for } & |\boldsymbol{c} \boldsymbol{\Phi} \boldsymbol{e}(k)| \geq \varepsilon \\
u(k-1) & \text { for } & |\boldsymbol{c} \boldsymbol{\Phi} \boldsymbol{e}(k)|<\varepsilon
\end{array} .\right.
$$

As the reference value $s_{r}(k)$ is generated a priori at every time instant $\{k\}$, its convergence to zero is not affected by the event-triggering algorithm. However, as the control signal is not updated at each periodic instant $k$, the actual trajectory of the system Equation (1) may deviate from the reference between instants $j$ and $j+1$. The conditions necessary to preserve the system's stability are discussed in the next section.

\subsection{Stability of the Event-Triggered System}

Having introduced a certain admissible state margin $\varepsilon$, we modify the reference trajectory and the trajectory following the reaching law. We require the system's represen- 
tative point to follow the reference position with accuracy to the disturbance $D(k)$ plus the admissible error margin. The reaching function, which ensures such a behaviour is

$$
s(k+1)=s_{r}(k+1)+D(k)+\phi(k),
$$

where $\phi(k)$ denotes the state error $c \boldsymbol{\Phi} \boldsymbol{e}(k)$ and $|\phi(k)| \leq \varepsilon$. On the other hand, the reference sliding variable profile must ensure convergence to the vicinity of the sliding plane despite the disturbance $D(k)$ and the state margin $\varepsilon$. Thus, the reaching law Equation (5) is transformed to

$$
s_{r}(k+1)=\left\{\begin{array}{cc}
(\alpha+\beta) s_{r}(k)-\alpha \beta s_{r}(k-1) & \text { for } \alpha \beta\left|s_{r}(k-1)\right|>2\left(D_{\max }+\varepsilon\right) \\
0 & \text { for } \alpha \beta\left|s_{r}(k-1)\right| \leq 2\left(D_{\max }+\varepsilon\right)
\end{array} .\right.
$$

Consequently, in the first stage of motion, the rate of change of the reference value satisfies

$$
\Delta s_{r}=\left|s_{r}(k)-s_{r}(k+1)\right|=\underbrace{[1-(\alpha+\beta)]}_{\langle 0 ; 1\rangle}\left|s_{r}(k)\right|+\underbrace{\alpha \beta\left|s_{r}(k-1)\right|}_{>2\left(D_{\max }+\varepsilon\right)} .
$$

Thus, for any $k \leq k_{0}$,

$$
\Delta s_{r}>2\left(D_{\max }+\varepsilon\right)
$$

and in the second stage of motion, for any $k \geq k_{0}+1, s_{r}(k)=0$. It may be easily shown that this modified reference trajectory ensures convergence of the system's trajectory to the vicinity of the sliding plane. Let us consider two consecutive state variable values, $s(k+1)$ and $s(k+2)$. For any $k<k_{0}$, the sliding variable rate of change may be expressed as

$$
|s(k+1)-s(k+2)|=\left|s_{r}(k+1)-s_{r}(k+2)+D(k)-D(k+1)+\phi(k)-\phi(k+1)\right| .
$$

Considering (30), one notices that

$$
|s(k+1)-s(k+2)|>0,
$$

so the system's trajectory converges to the vicinity of the sliding plane. On the other hand, for any $k \geq k_{0}+1, s_{r}(k)=0$. Therefore,

$$
|s(k)| \leq D_{\max }+\varepsilon
$$

for any $k \geq k_{0}+1$, which determines the width of the quasi-sliding mode band. Comparing the width of the quasi-sliding mode bands in Equations (22) and (33), it is visible that the application of the event-triggered control results in a band of greater radius, so it weakens the system's robustness. However, this is the price that must be paid for the reduction of the communication needs between the system's elements, thanks to aperiodic control signal updates.

\section{Simulation Example}

We conclude the above study with a simulation example. We design the eventtriggered quasi-sliding mode for a class of digitally controlled mechanical actuators. The discrete time system under consideration is described with

$$
\boldsymbol{\eta}(k+1)=\boldsymbol{\Phi} \boldsymbol{\eta}(k)+\boldsymbol{\Gamma}[u(k)+f(k)],
$$

where the state matrix and input distribution vector are

$$
\boldsymbol{\Phi}=\left[\begin{array}{ll}
1 & 0.8639 \\
0 & 0.7408
\end{array}\right] \quad \text { and } \quad \boldsymbol{\Gamma}=\left[\begin{array}{l}
0.4535 \\
0.8639
\end{array}\right]
$$


and the sampling period $T=1$. The initial state vector is $\eta_{0}=\left[\begin{array}{lll}250 & 0\end{array}\right]^{\mathrm{T}}$. The system is subject to the disturbance $f(k)$ changing sinusoidally between \pm 1 . First, we are going to compare the results of the reference trajectory-based sliding mode control with and without event-triggering. In the next part, we will compare the event-triggered algorithm with a conventional quasi-sliding mode control introduced in [15].

We begin the design of the sliding mode with a selection of the sliding plane, whose choice determines the stability of the control system. Therefore, we consider the plant (34) whose dynamics are bounded to the sliding surface Equation (3). With the application of the control Equation (17), for any $k \geq k_{0}$, the closed-loop system becomes

$$
\boldsymbol{\eta}(k+1)=\left[1-\boldsymbol{\Gamma}(\boldsymbol{c} \boldsymbol{\Gamma})^{-1} \boldsymbol{c}\right] \boldsymbol{\Phi} \boldsymbol{\eta}(k)+\boldsymbol{\Gamma} f(k) .
$$

Thus, in order to guarantee the stability of the control system, the eigenvalues of the matrix $\left[1-\Gamma(c \Gamma)^{-1} c\right] \Phi$ must be located within the unit circle. For that purpose we choose $c=[10.475]$ so that both eigenvalues are equal to zero. With this choice of vector $c$, the maximum disturbance influence on the sliding variable is $D_{\max }=0.864$.

We will compare the performance of the system with and without event-triggering. Therefore, we generate the reference sliding variable profile with the reaching laws Equations (5) and (28), respectively. We choose parameters $\alpha=\beta=0.5$ and the error margin for the event-triggered algorithm is $\varepsilon=0.3$. The evolution of the reference $s_{r}(k)$ is presented in Figure 1.

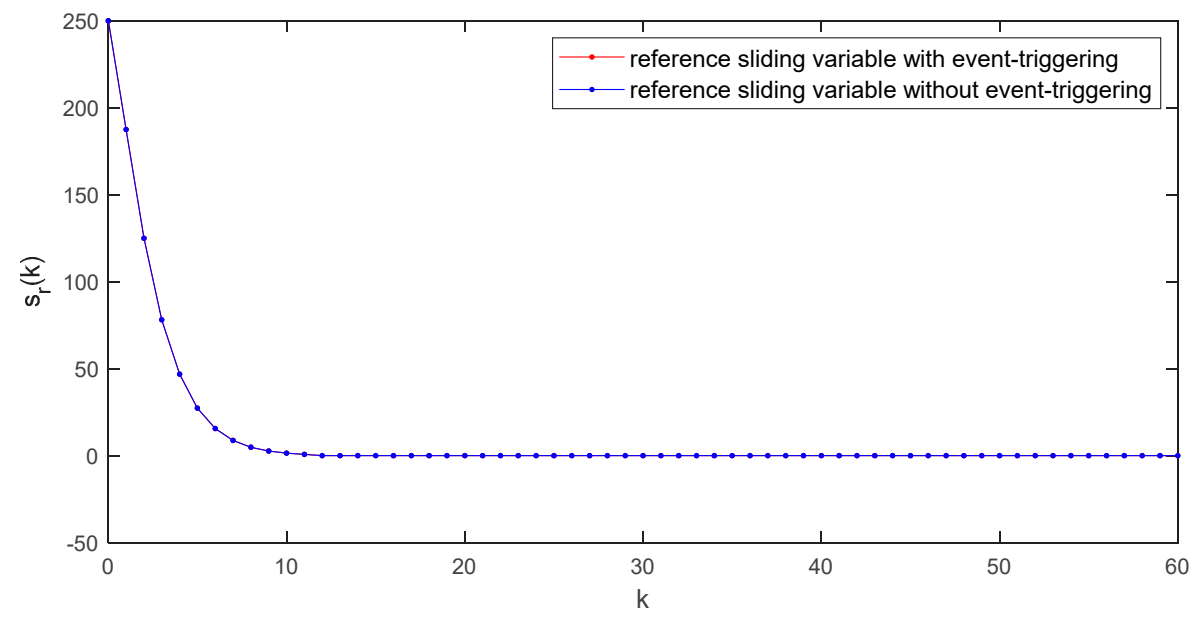

Figure 1. Reference sliding variable with and without event-triggering.

As shown in Figure 1, the novel reaching law Equation (5) ensures monotonic convergence to the sliding hyperplane and a nonswitching movement along this hyperplane. The reference sliding variable equals zero for any $k \geq 12$. In the next step, the generated reference profile will be used to control the perturbed system Equation (34). We apply the control signals Equations (17) and (26), respectively. In the event-triggered case, we aim to reduce the communication between elements of the system and reduce control delays. Therefore, the control signal is updated each time the triggering condition Equation (25) is met. The control input for both considered cases is presented in Figures 2 and 3 and the resulting sliding variable evolution is presented in Figures 4 and 5. Finally, Figure 6 presents the evolution of the state variables. 


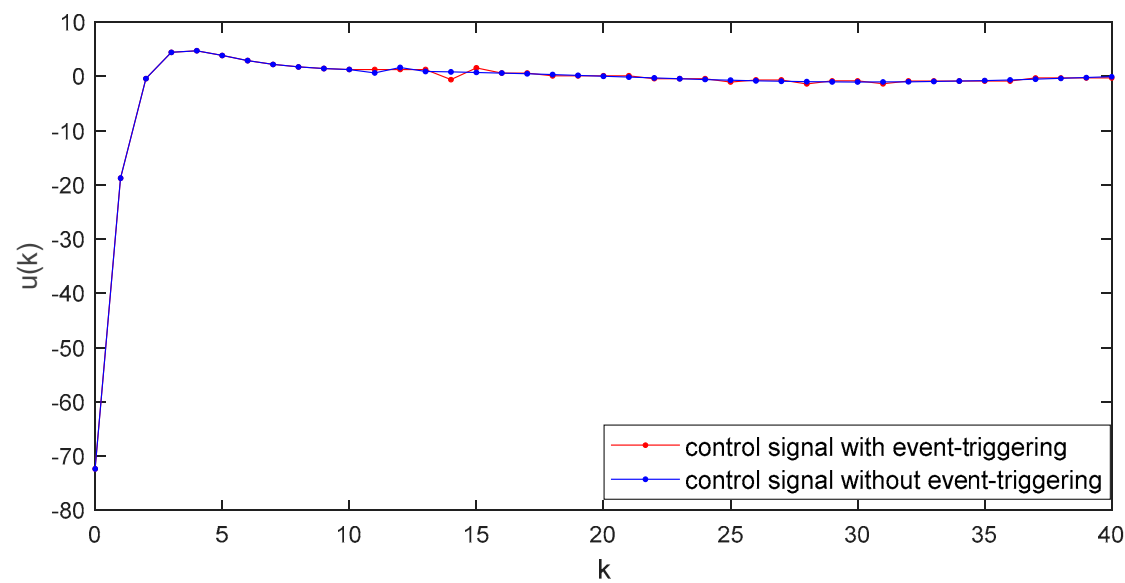

Figure 2. Control signal.

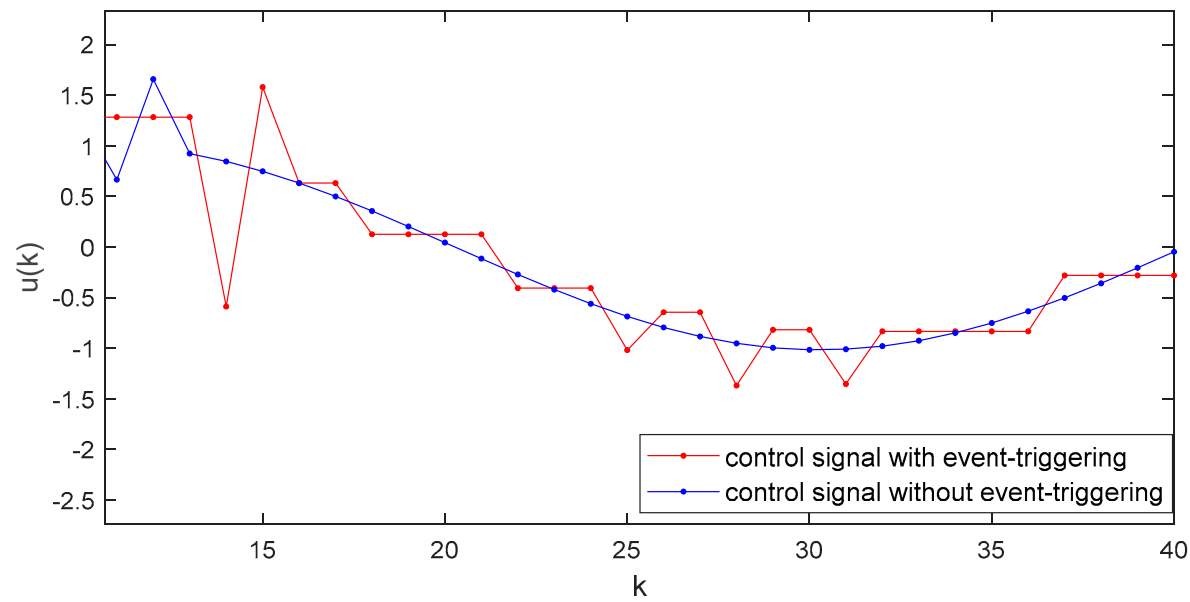

Figure 3. Control signal (zoomed).

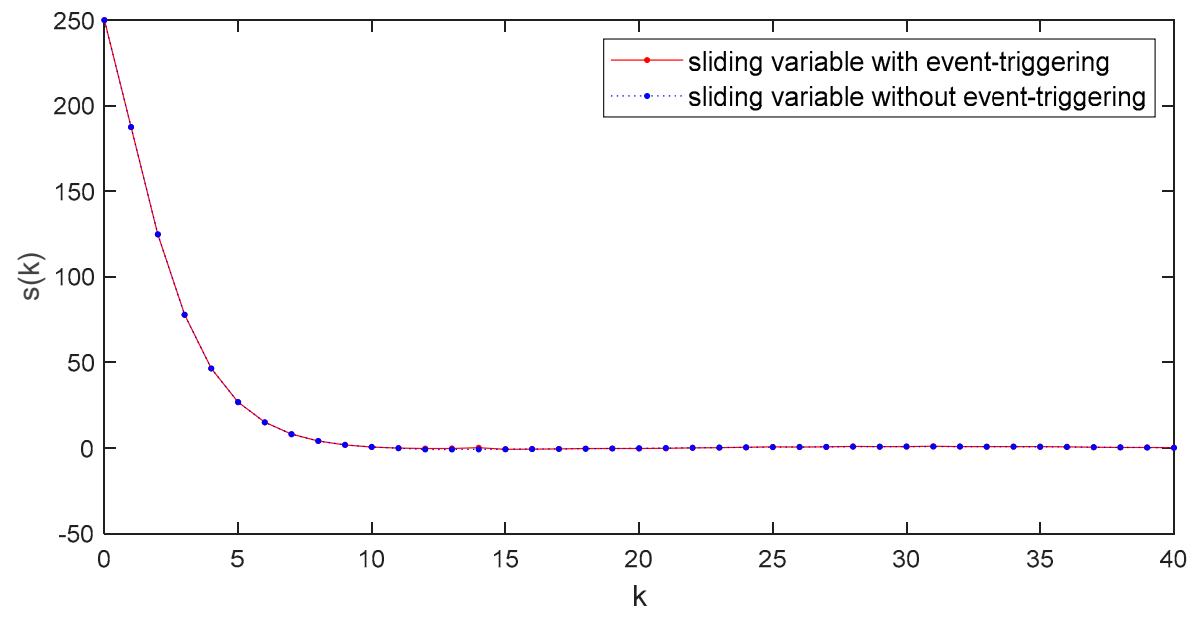

Figure 4. The sliding variable profile. 


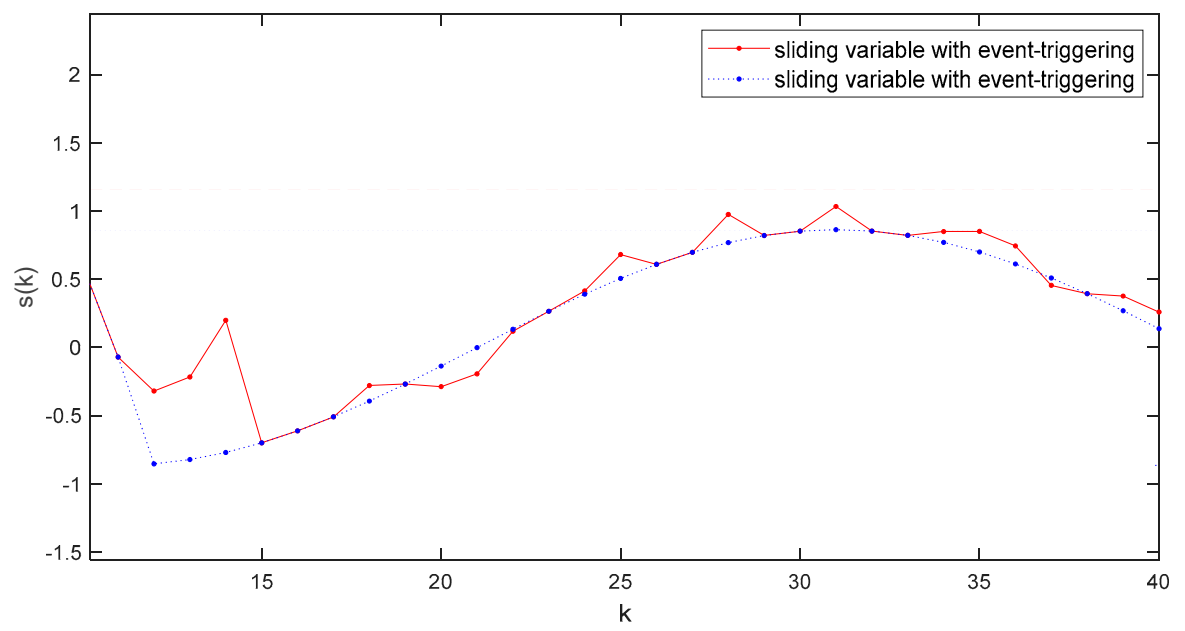

Figure 5. The sliding variable in the sliding phase.
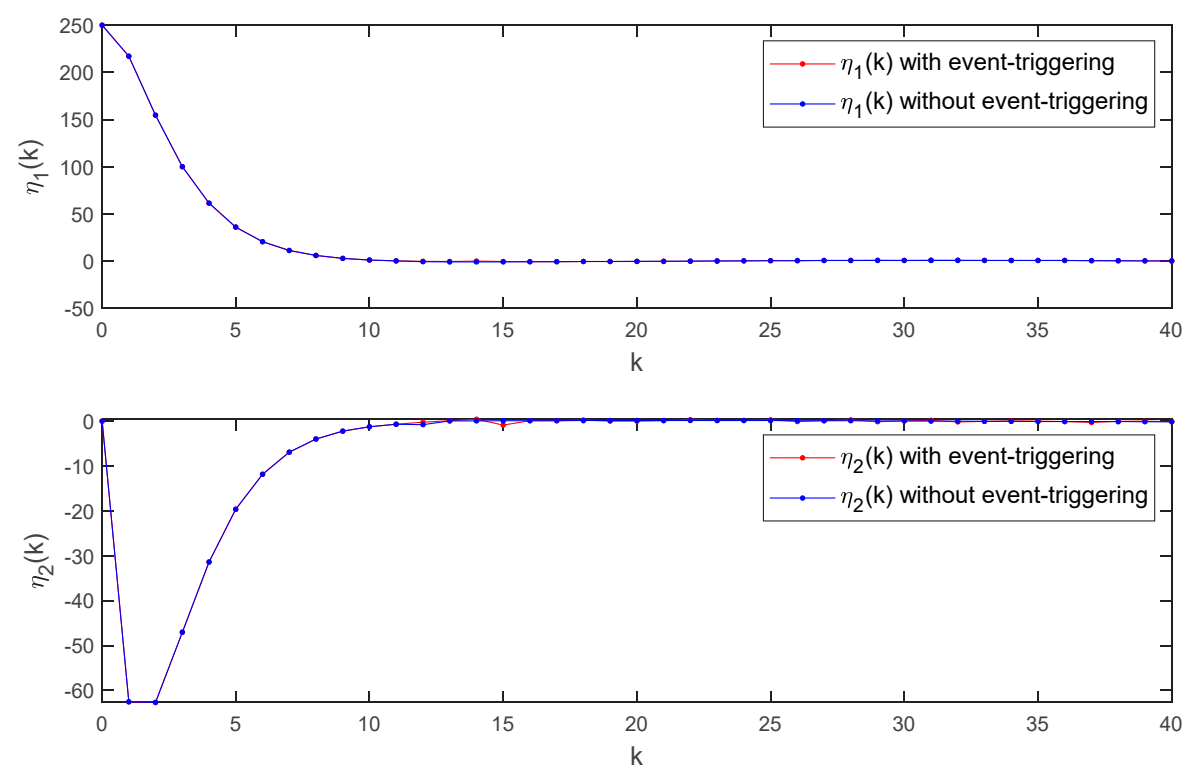

Figure 6. The state variables.

From the above figures, one may easily notice that, although the control signal is not updated at every periodic instant in the event-triggered control scheme, a stable quasi-sliding mode is ensured. The sliding phase begins at instant $k=12$. For any $k \geq 12$ the sliding variable $s(k)$ belongs to the interval $\left\langle-D_{\max }-\varepsilon, D_{\max }+\varepsilon\right\rangle$, which in this case equals $\langle-1.164,1.164\rangle$. On the other hand, the strategy without event-triggering results in the quasi-sliding mode band of $\left\langle-D_{\max }, D_{\max }\right\rangle$, which equals $\langle-0.864,0.864\rangle$. It may be noticed that the introduction of the event-triggering scheme caused only a small enlargement of the quasi-sliding mode bandwidth. Moreover, the proposed control strategy does not require switching the sign of the sliding variable in each step, which eliminates the problem of chattering and reduces the control effort in the sliding phase.

In the second part of our simulations, we decided to compare our event-triggered control algorithm with a conventional quasi-sliding mode control proposed in [15]. Therefore, according to [15], we applied the following control signal

$$
u(k)=-(c c \Gamma)^{-1} c \Phi \eta(k),
$$

which drives the sliding variable to the vicinity of the sliding surface in one step. The comparison of the applied control signals is presented in Figure 7. 


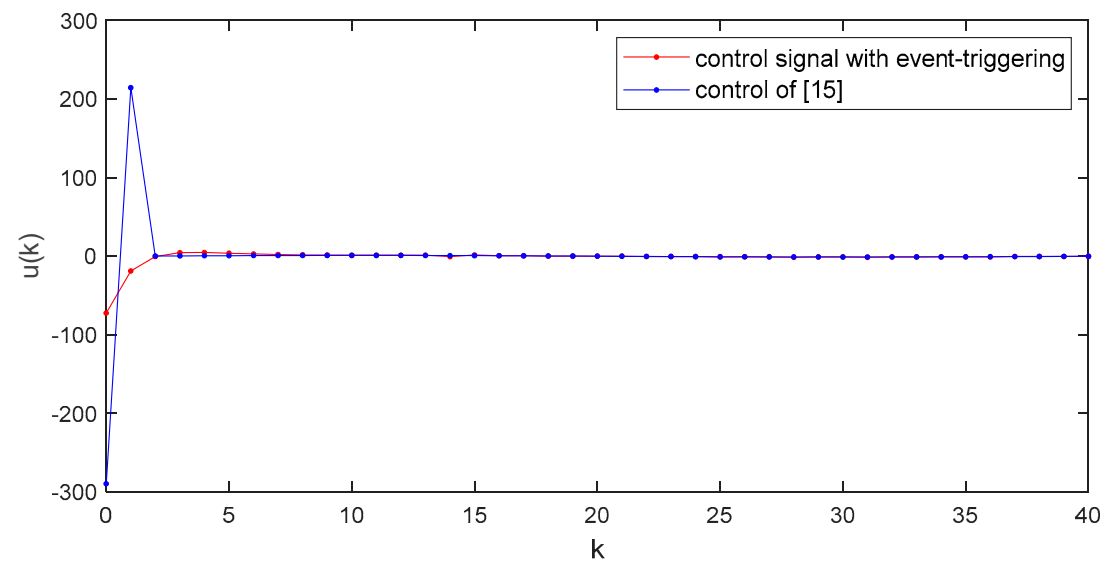

Figure 7. Comparison of the control signals.

It may be easily noticed from Figure 7 that the conventional control strategy requires much larger control input at the beginning of the motion. The strategy proposed in this paper significantly reduces the control effort, which directly results in energy saving. However, this is obtained with much slower convergence to the sliding surface, shown in Figures 8 and 9 .

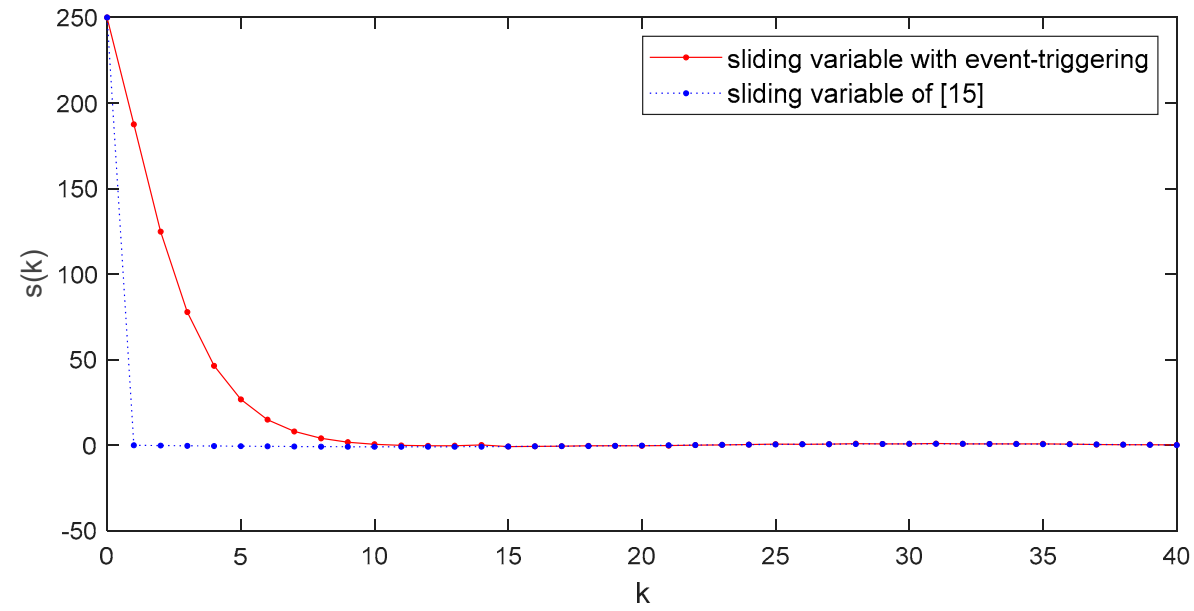

Figure 8. The sliding variable for both control strategies.

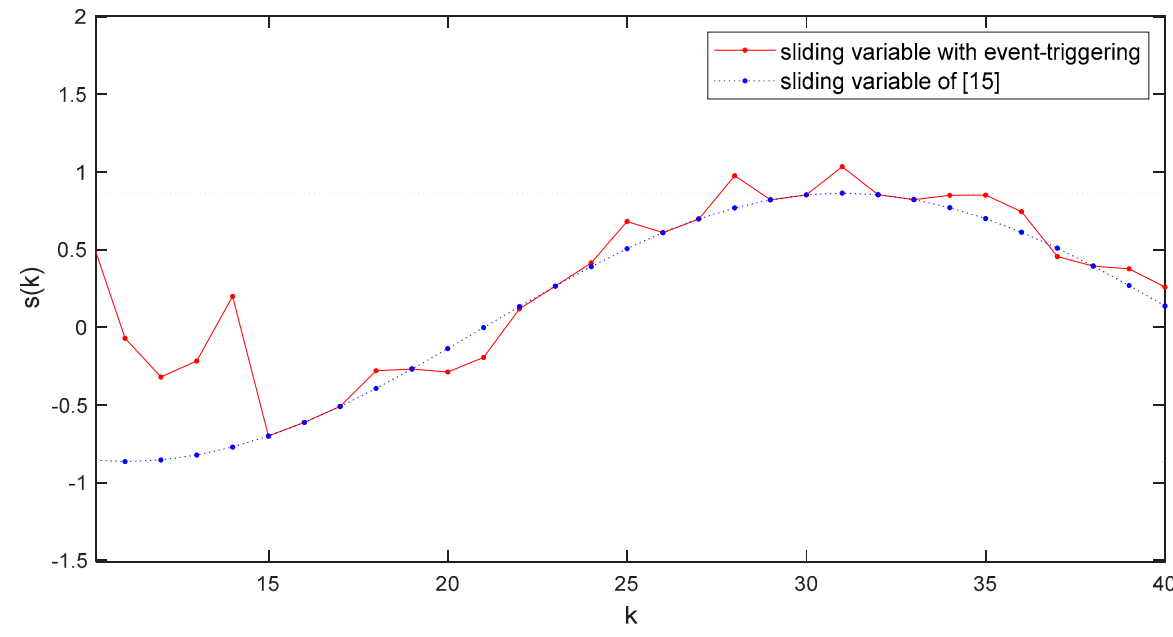

Figure 9. Sliding variable in the sliding phase. 
Finally, Figure 10 presents the evolution of the state variables. It is clear that faster convergence enforces higher state variables values, which may be undesirable in mechanical or electrical applications.
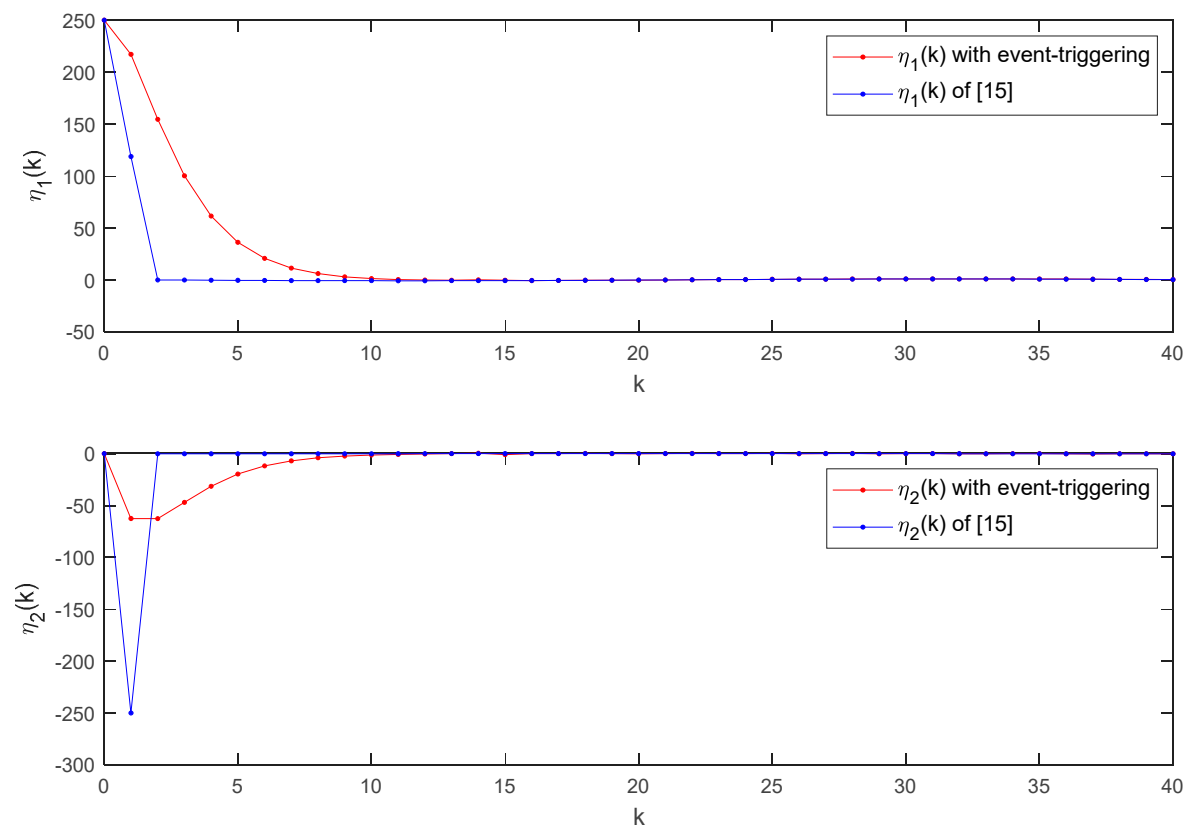

Figure 10. The state variables.

\section{Final Remarks}

This study discussed the sliding mode control design for discrete time perturbed systems. We introduced a trajectory tracking control strategy, which limits the impact of external disturbances to one sampling step only. First, we presented a novel reaching law, applied to generate the reference profile of the sliding variable. The proposed reaching law ensures convergence to the sliding surface and a non-switching motion along this surface. Having obtained the reference trajectory, we proposed a reference trajectory tracking control law for the actual plant. It is shown that the proposed algorithm provides stability to the closed-loop system and improves its robustness by minimizing the perturbation effects. Moreover, in the further part of the study, we modified our control strategy by introducing an event-triggering mechanism. In order to reduce the need for communication between sensor and actuator ends of the control system, we propose to update the control signal only when a certain stability condition is violated instead of updating it periodically in each discrete time step. Although such a modification slightly enlarges the quasi-sliding mode bandwidth, it may bring numerous benefits such as lower bandwidth consumption for communication, delays reduction, increased computational efficiency, and energy saving. The idea presented in this study is quite versatile and may be easily extended by the application of different reference trajectory profiles. Our future research plans include testing different reaching laws for the reference sliding variable and practical application of the method.

Author Contributions: Conceptualization, A.B. and K.A.; methodology, A.B. and K.A.; validation, A.B. and K.A.; formal analysis, K.A.; investigation, K.A.; writing—original draft preparation, K.A.; writing-review and editing, A.B.; visualization, K.A.; supervision, A.B. All authors have read and agreed to the published version of the manuscript.

Funding: This research received no external funding.

Conflicts of Interest: The authors declare no conflict of interest. 


\section{References}

1. Emelyanov, S.V. Variable Structure Control Systems; Nauka: Moscow, Russia, 1967.

2. Utkin, V.I. Variable structure systems with sliding modes. IEEE Trans. Automat. Contr. 1977, 22, 212-222. [CrossRef]

3. Draženović, B. The invariance conditions in variable structure systems. Automatica 1969, 5, 287-295. [CrossRef]

4. Bartolini, G.; Zolezzi, T. Variable structure systems nonlinear in the control law. IEEE Trans. Automat. Contr. 1985, 30, 681-684. [CrossRef]

5. DeCarlo, R.A.; Żak, S.; Matthews, G. Variable structure control of nonlinear multivariable systems: A tutorial. Proc. IEEE 1988, 76, 212-232. [CrossRef]

6. Utkin, V.I. Sliding Modes in Control and Optimization; Springer: Berlin, Germany, 1992.

7. Levant, A. Sliding order and sliding accuracy in sliding mode control. Int. J. Contr. 1993, 58, 1247-1263. [CrossRef]

8. Gao, W.B.; Hung, J.C. Variable structure control of nonlinear systems: A new approach. IEEE Trans. Ind. Electron. 1993, 40, 45-55.

9. Edwards, C.; Spurgeon, S. Sliding Mode Control: Theory and Applications; Taylor \& Francis: London, UK, 1998.

10. Mobayen, S.; Bayat, F.; Lai, C.-C.; Taheri, A.; Fekih, A. Adaptive global sliding mode controller design for perturbed DC-DC buck converters. Energies 2021, 14, 1249. [CrossRef]

11. Oucheriah, S.; Guo, L. PWM-based adaptive sliding-mode control for boost DC-DC converters. IEEE Trans. Ind. Electron. 2013, 60, 3291-3294. [CrossRef]

12. Shtessel, Y.; Lee, Y.J. New approach to chattering analysis in systems with sliding modes. In Proceedings of the 35th Conference on Decision and Control, Kobe, Japan, 13 December 1996; pp. 4014-4019.

13. Milosavljević, C. General conditions for the existence of a quasi-sliding mode on the switching hyperplane in discrete variable structure systems. Automat. Remote Contr. 1985, 46, 3307-3314.

14. Gao, W.B.; Wang, Y.; Homaifa, A. Discrete-time variable structure control systems. IEEE Trans. Ind. Electron. 1995, 42, $2117-2122$.

15. Utkin, V.I.; Drakunov, S.V. On discrete-time sliding modes. IFAC Proc. Vol. 1989, 22, 3273-3278.

16. Kaynak, O.; Denker, A. Discrete time sliding mode control in the presence of system uncertainty. Int. J. Contr. 1993, 57, 51177-51189. [CrossRef]

17. Bartolini, G.; Ferrara, A.; Utkin, V.I. Adaptive sliding mode control in discrete-time systems. Automatica 1995, 31, 5769-5773. [CrossRef]

18. Golo, G.; Milosavljević, C. Robust discrete-time chattering free sliding mode control. Syst. Contr. Lett. 2000, 41, 19-28. [CrossRef]

19. Niu, Y.; Ho, D.W.C.; Wang, Z. Improved sliding mode control for discrete-time systems via reaching law. IET Proc. Contr. Theory Appl. 2010, 4, 2245-2251. [CrossRef]

20. Chakrabarty, S.; Bandyopadhyay, B. Minimum ultimate band design of discrete sliding mode control. Asian J. Contr. 2015, 17, 51189-51897. [CrossRef]

21. Chakrabarty, S.; Bandyopadhyay, B. A generalized reaching law with different convergence rates. Automatica 2016, 63, 34-37. [CrossRef]

22. Ma, H.; Wu, J.; Xiong, Z. A novel exponential reaching law of discrete-time sliding-mode control. IEEE Trans. Ind. Electron. 2017, 64, 63840-63850. [CrossRef]

23. Cunha, J.P.V.S.; Hsu, L.; Costa, R.R.; Lizarralde, F. Output-feedback model-reference sliding mode control of uncertain multivariable systems. IEEE Trans. Automat. Contr. 2003, 48, 2245-2250. [CrossRef]

24. Bartoszewicz, A.; Adamiak, K. Model reference discrete-time variable structure control. Int. J. Adapt. Contr. Signal Process. 2018, 32, 1440-1452. [CrossRef]

25. Bandyopadhyay, B.; Behera, A.K. Event-Triggered Sliding Mode Control, Studies in Systems, Decision and Control 139; Springer: Cham, Switzerland, 2018.

26. Behera, A.K.; Bandyopadhyay, B.; Reger, J. Discrete event-triggered sliding mode control with fast output sampling feedback. In Proceedings of the 2016 14th International Workshop on Variable Structure Systems (VSS), Nanjing, China, 1-4 June 2016; pp. 148-153.

27. Behera, A.K.; Bandyopadhyay, B.; Yu, X. Periodic event-triggered sliding mode control. Automatica 2018, 96, 61-72. [CrossRef]

28. Shah, D.; Santos, M.; Chaoui, H.; Justo, J. Event-triggered non-switching networked sliding mode control for active suspension system with random actuation network delay. IEEE Trans. Intel. Transport. Sys. 2021. [CrossRef]

29. Mehta, A.; Bandyopadhyay, B. Emerging Trends in Sliding Mode Control, Studies in Systems, Decision and Control 318; Springer: Singapore, 2021. 